EPJ Web of Conferences 18, 03001 (2011)

DOI: $10.1051 /$ epjconf/20111803001

(C) Owned by the authors, published by EDP Sciences, 2011

\title{
Interstellar dust within the life cycle of the interstellar medium
}

\author{
K. Demyk ${ }^{1,2, a}$ \\ ${ }^{1}$ Université de Toulouse, UPS-OMP, IRAP, Toulouse, France \\ ${ }^{2}$ CNRS, IRAP, 9 Av. colonel Roche, BP. 44346, 31028 Toulouse Cedex 4, France
}

\begin{abstract}
Cosmic dust is omnipresent in the Universe. Its presence influences the evolution of the astronomical objects which in turn modify its physical and chemical properties. The nature of cosmic dust, its intimate coupling with its environment, constitute a rich field of research based on observations, modelling and experimental work. This review presents the observations of the different components of interstellar dust and discusses their evolution during the life cycle of the interstellar medium.
\end{abstract}

\section{INTRODUCTION}

Interstellar dust grains are found everywhere in the Universe: in the Solar System, around stars at all evolutionary stages, in interstellar clouds of all kind, in galaxies and in the intergalactic medium. Cosmic dust is intimately mixed with the gas-phase and represents about $1 \%$ of the gas (in mass) in our Galaxy. The interstellar extinction and the emission of diffuse interstellar clouds is reproduced by three dust components: a population of large grains, the BGs (Big Grains, $\sim 10-500 \mathrm{~nm}$ ) made of silicate and a refractory mantle, a population of carbonaceous nanograins, the VSGs (Very Small Grains, 1-10 nm) and a population of macro-molecules the PAHs (Polycyclic Aromatic Hydrocarbons) [1]. These three components are more or less abundant in the diverse astrophysical environments reflecting the coupling of dust with the environment and its evolution according to the physical and dynamical conditions.

The life cycle of cosmic matter is closely related to the cycle of the interstellar medium (ISM) and of our Galaxy. Dust is mainly form within the atmosphere and circumstellar shells surrounding dying stars. It is expelled in the ISM by stellar outflows and transit in the different phases of the ISM where it is submitted to various physical and chemical processes that either destroy it or change its nature (size, structure, composition, porosity...). When a molecular cloud collapse to form new stars, the interstellar dust grains are incorporated into the protostellar object in which the survival grains pursue their evolution, into proto-planetary disks and stellar systems. At their turn, these stars, at the end of their life will produce new dust grains.

Interstellar dust was extensively studied via the observations from the instruments on-board the space-missions IRAS (InfraRed Astronomical Satellite, 1983), ISO (Infrared Space Observatory, 1995-1998) and Spitzer (2003-2005), free from the atmosphere opacity in the infrared, which gave tremendous amount of data. While these missions were observing in the mid- and far-infrared spectral range $(3-200 \mu \mathrm{m})$, the satellite Herschel, launched in May 2009, opens the still unexplored spectral range between infrared and ground-based radio observations $(60-672 \mu \mathrm{m}, 5 \mathrm{THz}-480 \mathrm{GHz})$. The spectrophotometers on-board these satellites allow to map with an increasing angular resolution the spatial distribution of the different dust components, at the Galaxy scale, but also in specific objects.

ae-mail: karine.demyk@irap.omp.eu

This is an Open Access article distributed under the terms of the Creative Commons Attribution-Noncommercial License 3.0, which permits unrestricted use, distribution, and reproduction in any noncommercial medium, provided the original work is properly cited. 
The spectroscopic observations reveal the dust (and gas) composition via the identification of the numerous spectral features observed in emission or in absorption.

To interpret the observational data, strong experimental efforts are made in order to synthesise analogs of the interstellar dust and to simulate their evolution. This domain of astrochemistry implies a coupling between astrophysicists and physicists, chemists, and mineralogists. For the last 40 years, numerous experimental setups have been developed all over the world to produce analogs of silicate dust, ices, and carbonaceous dust and to study their spectroscopic properties. Their formation, destruction and evolution caused by their interaction with photons, gas phase molecules, and cosmic rays are studied under physical conditions as close as feasible to those of the ISM (low pressure and temperature). This approach has been successful in identifying many of the major interstellar dust components such as the ices and the silicate dust. However there is still a lot to do to understand the dust evolution.

This non exhaustive review addresses different aspects of the studies of interstellar dust. The different dust components, their physical nature and composition as revealed by spectroscopic observations are presented Section 2. Their formation is addressed in Sect 3. The dust evolution during the ISM cycle is presented in Sect. 4. Some open questions concluded this review in Sect. 5.

\section{OBSERVATION OF INTERSTELLAR DUST: NATURE AND COMPOSITION}

The physical and chemical nature of interstellar dust is mainly inferred (i) from spectroscopic studies of the light absorbed and scattered by the dust grains, i.e., of the so-called extinction curve and of the light emitted by the dust grains and (ii) from the depletion of heavy elements in the gas phase. The depletion of the elements in the interstellar medium (ISM) is defined as the abundance of the elements missing from the gas phase with respect to a given reference abundance system. The missing atoms are assumed to be bound into the dust grains. The major dust constituents are $\mathrm{C}$ and $\mathrm{O}$ (more than $\geq 300 \mathrm{ppm})$ followed by $\mathrm{Mg}, \mathrm{Si}$, and $\mathrm{Fe}(\sim 30 \mathrm{ppm})$, minor components $\mathrm{Na}, \mathrm{Al}, \mathrm{Ca}, \mathrm{Ni}(\sim 3 \mathrm{ppm})$ and trace components K, Ti, Cr, Mn, Co ( $\sim 0.1-0.3 \mathrm{ppm})[2]$.

\subsection{The refractory oxygen rich dust: Silicates and oxides}

In the visible, the $\lambda^{-1}$ dependence of the extinction curve indicates the existence of a population of grain of the same size as the wavelength, i.e., $\sim 0.1-0.5 \mu \mathrm{m}$. Therefore, this dust component is frequently referred to as the "big grains" (BG). This grain population is ubiquitous, present around stars at all evolutionary stages, in interstellar clouds, in galaxies. The big grains are in thermal equilibrium with the interstellar radiation field (ISRF). In the interstellar medium, because of their low temperature $(\mathrm{T} \leq 30 \mathrm{~K})$, they dominate the emission of cold interstellar clouds in the submillimetric domain (Fig. 1). The big grains are responsible for various spectral bands observed in the mid-infrared $(8-70 \mu \mathrm{m})$ in absorption and/or in emission in all types of astronomical environments (Table 1). These features are due to the vibration of chemical bonds into the grain material, consequently, their study give access to the composition and structure of the dust.

The refractory grains are mainly composed of silicates of olivine $\left(\mathrm{Mg}_{2 x} \mathrm{Fe}_{2-2 x} \mathrm{SiO}_{4}\right)$ and pyroxene type $\left(\mathrm{Mg}_{y} \mathrm{Fe}_{1-y} \mathrm{SiO}_{3}\right)$. They are responsible for the broad spectral features observed at $\sim 9.7 \mu \mathrm{m}$ ( $\mathrm{Si}-\mathrm{O}$ stretching mode) and $\sim 18 \mu \mathrm{m}$ (O-Si-O bending mode) in absorption or in emission in many astronomical objects. Most of the interstellar silicates are amorphous as shown by the broadness and structure-less shape of these two bands (Fig. 2) and their exact composition is thus difficult to determine. However, one of the main results from the ISO observatory was the discovery, in some specific astronomical environments, of crystalline silicates that exhibit sharp substructures in the vibrational modes and numerous bands observed in emission in the 20-70 $\mu \mathrm{m}$ spectral range (Figs. 3, [4, 5]). Thanks to these sharp bands, crystalline silicates are well-identified and their composition well-constrained. This 


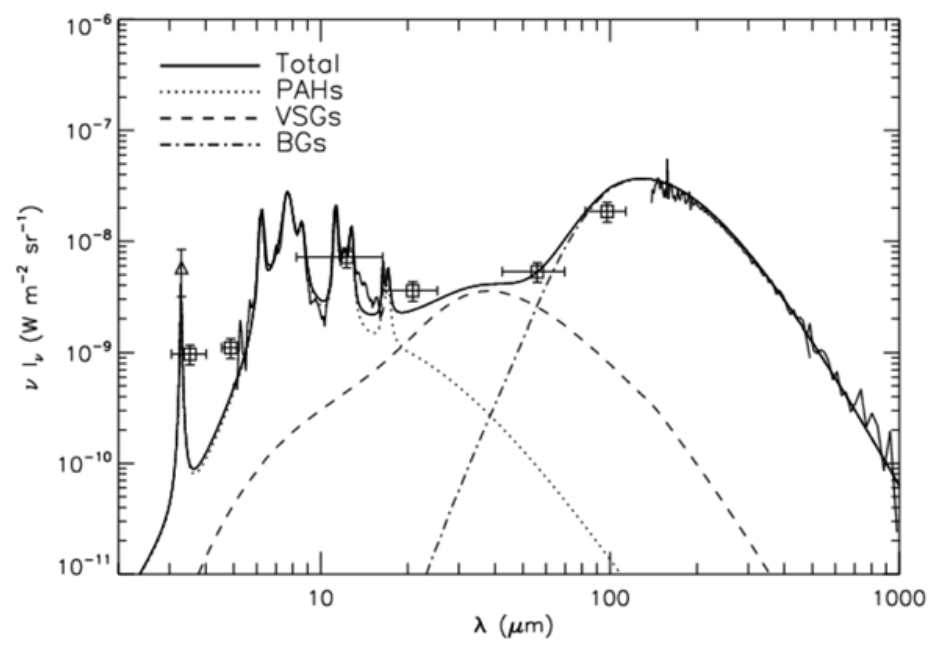

Figure 1. Emission spectrum for the diffuse interstellar medium as observed by ISOCAM-CVF, DIRBE and FIRAS and modelled for $\mathrm{N}_{\mathrm{H}}=10^{20} \mathrm{~cm}^{-2}$. The solid line is the dust model composed of three dust components: the Big Grains (dotted-dashed line), the Very Small Grains (dashed line) and the PAHs (dotted line). The observations are from the ISO and COBE satellites (see for [3] details). The figure is taken from [3].

important finding raised many questions on the evolutionary cycle of dust which are still debated (see Sect. 4.3).

In addition to silicates, oxides and sulphides are present. They are more difficult to study as their vibrational modes are broad and blended with the dominating silicate infrared features. Highly refractory oxides such as $\mathrm{TiO}_{2}, \mathrm{Al}_{2} \mathrm{O}_{3}$ or $\mathrm{Al}_{2} \mathrm{TiO}_{5}$, which are believed to be the nucleation seeds on which the silicates grains grow (see Sect. 3) and may therefore constitute the core of the silicate grains, are proposed to be the carriers of bands observed at $13 \mu \mathrm{m}, 19.5$ and $28 \mu \mathrm{m}$ [6]. Magnesium and iron oxides observed in the $\sim 18-22 \mu \mathrm{m}$ range may be present as a distinct grain population as suggested by the study of the depletion of heavy elements in line of sights toward cold and warm clouds, the destruction of Mg-rich grains being more efficient than Fe-rich grains in warm clouds [7]. Although common in cometary grains, sulphides, which are proposed to explain various emission lines in interstellar spectra, are not yet firmly detected in interstellar grains [8].

\subsection{The volatile oxygen rich dust: Interstellar ices}

In dense environments the atoms and molecules in the gas phase freeze onto the grains and form an icy mantle surrounding them. Interstellar ices are observed in circumstellar environments around OH/IR stars, field stars and protostars. Their composition depends on the object in which they are formed and on the physico-chemical processes they underwent. The richest ice mantles contain neutral molecules such as $\mathrm{H}_{2} \mathrm{O}, \mathrm{CO}_{2}, \mathrm{CO}, \mathrm{CH}_{3} \mathrm{OH}, \mathrm{CH}_{4}, \mathrm{H}_{2} \mathrm{CO}$ but also ions such as $\mathrm{OCN}^{-}$and $\mathrm{NH}_{4}^{+}$(e.g., [9], Fig. 2, Table 2). Other more complex species are probably present in the ice mantles. However, unless they have strong oscillator strength, their identification in interstellar spectra is difficult because of their low abundance and because of blending problems with lines from other dominant ice species.

The analysis of the line profiles gives important information on the ice structure and on the molecular interaction between the ice constituents. The $\mathrm{CO}$ and $\mathrm{CO}_{2}$ band profiles are affected by the overall composition of ice and allow to trace segregation within the ice mantle of polar (dominated by molecules with strong $\mathrm{H}$-bonds such as $\mathrm{H}_{2} \mathrm{O}$ and $\mathrm{CH}_{3} \mathrm{OH}$ and apolar ices ([11, 12], [9] and reference therein). The shape of the $\mathrm{OH}$ stretching mode of $\mathrm{H}_{2} \mathrm{O}$ at $\sim 3 \mu$ m contains many information $(i)$ on the ice crystallinity 
EPJ Web of Conferences

Table 1. List of the main observed bands attributed to silicate dust. Adapted from [4].

\begin{tabular}{lll}
\hline Observed bands & Assignments & composition \\
\hline $9.7 \mu \mathrm{m}$ & $\begin{array}{l}\text { amorphous silicate } \\
\text { amorphous silicate }\end{array}$ & $\begin{array}{l}\mathrm{Si}-\mathrm{O} \text { stretching mode } \\
\mathrm{O}-\mathrm{Si}-\mathrm{O} \text { bending mode }\end{array}$ \\
\hline $9.8 \mu \mathrm{m}$ & forsterite + enstatite & $\mathrm{Mg}_{2} \mathrm{SiO}_{4}+\mathrm{MgSiO}_{3}$ \\
$10.7 \mu \mathrm{m}$ & enstatite & $\mathrm{MgSiO}_{3}$ \\
$11.4 \mu \mathrm{m}$ & forsterite + diopside & $\mathrm{Mg}_{2} \mathrm{SiO}_{4}+\mathrm{CaMgSi}_{2} \mathrm{O}_{6}$ \\
$15.2 \mu \mathrm{m}$ & enstatite & $\mathrm{MgSiO}_{3}$ \\
$16.2 \mu \mathrm{m}$ & forsterite & $\mathrm{Mg}_{2} \mathrm{SiO}_{4}$ \\
$17.5 \mu \mathrm{m}$ & enstatite & $\mathrm{MgSiO}_{3}$ \\
$18 \mu \mathrm{m}$ & enstatite + forsterite & $\mathrm{MgSiO}_{3}+\mathrm{Mg}_{2} \mathrm{SiO}_{4}$ \\
$19.5 \mu \mathrm{m}$ & enstatite + forsterite & $\mathrm{MgSiO}_{3}+\mathrm{Mg}_{2} \mathrm{SiO}_{4}$ \\
$23 \mu \mathrm{m}$ & enstatite & $\mathrm{MgSiO}_{3}$ \\
$23.7 \mu \mathrm{m}$ & forsterite & $\mathrm{Mg}_{2} \mathrm{SiO}_{4}$ \\
$27.6 \mu \mathrm{m}$ & forsterite & $\mathrm{Mg}_{2} \mathrm{SiO}_{4}$ \\
$28.2 \mu \mathrm{m}$ & enstatite & $\mathrm{MgSiO}_{3}$ \\
$33.6 \mu \mathrm{m}$ & forsterite & $\mathrm{Mg}_{2} \mathrm{SiO}_{4}$ \\
$39.8 \mu \mathrm{m}$ & diopside & $\mathrm{CaMgSi}_{2} \mathrm{O}_{6}$ \\
$40.5 \mu \mathrm{m}$ & enstatite & $\mathrm{MgSiO}_{3}$ \\
$43 \mu \mathrm{m}$ & enstatite + water ice & $\mathrm{MgSiO}_{3}+\mathrm{cryst}_{2} \mathrm{O}$ ice \\
$43.8 \mu \mathrm{m}$ & enstatite & $\mathrm{MgSiO}_{3}$ \\
$69 \mu \mathrm{m}$ & forsterite & $\mathrm{Mg}_{2} \mathrm{SiO}_{4}$ \\
\hline & & \\
\hline & & \\
\hline & &
\end{tabular}

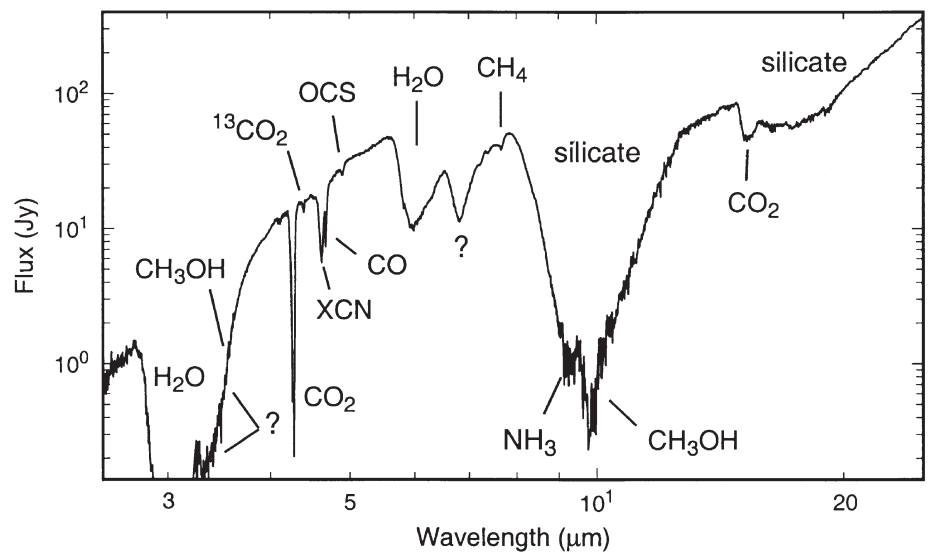

Figure 2. ISO-SWS spectrum of the protostar W33A. The identification of the main ice species spectral features and of the amorphous silicate modes at 9.7 and $18 \mu \mathrm{m}$ are indicated on the figure. Adapted from [10].

as the line is sharp when the water ice is crystalline as observed in OH/IR stars whereas it is broad and structureless in amorphous ices [13], (ii) on the grain size as the line becomes asymmetric due to the effect of scattering when the grains grow [14]. The band at $3.47 \mu \mathrm{m}$, correlated with $\mathrm{H}_{2} \mathrm{O}$ ice, traces interaction between $\mathrm{NH}_{3}$ and the $\mathrm{OH}$ bond of $\mathrm{H}_{2} \mathrm{O}$ and in ammonia hydrates [14]. Interactions 

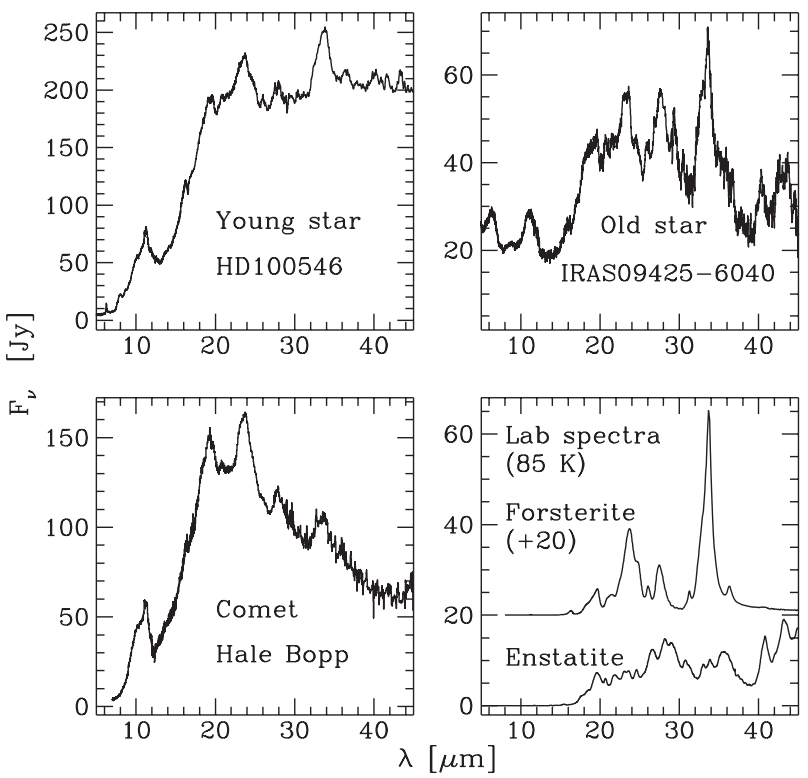

Figure 3. ISO-SWS spectrum of sources exhibiting crystalline silicate features: young star (upper left), evolved star (upper right) and comet (lower left). The lower right panel shows laboratory spectra of enstatite $\left(\mathrm{MgSiO}_{3}\right)$ and forsterite $\left(\mathrm{Mg}_{2} \mathrm{SiO}_{4}\right)$. The figure is taken from [5].

Table 2. Composition of ices in various environments.

\begin{tabular}{llccccc}
\hline Species & $\begin{array}{l}\text { IR active } \\
\text { vibration modes } \\
(\mu \mathrm{m})\end{array}$ & $\begin{array}{c}\text { Quiescent } \\
\text { clouds }\end{array}$ & $\begin{array}{c}\text { Low-mass } \\
\text { YSOs }\end{array}$ & $\begin{array}{c}\text { Antermediate-mass } \\
\text { YSOs }\end{array}$ & $\begin{array}{c}\text { High-mass YSOs } \\
\text { low processing strong processing }\end{array}$ \\
\hline $\mathrm{H}$ & & & & & & \\
$\mathrm{HO}$ & $3.05,6.02,13.15$ & 100 & 100 & 100 & 100 & 100 \\
$\mathrm{CO}$ & 4.67 & $\sim 25$ & $\sim 5$ & $\sim 18-50$ & $\sim 3-8$ & $\sim 8-17$ \\
$\mathrm{NH}_{3}$ & $4.26,15.15$ & $\sim 25$ & $\sim 20$ & $\sim 30-35$ & $\sim 7-22$ & $\sim 13-23$ \\
& $2.96-3.11$, & $\leq 10$ & $\leq 5$ & $\leq 5$ & $\leq 5$ & $\sim 15$ \\
$\mathrm{CH}_{3} \mathrm{OH}$ & $5.97,9.34$ & & & & & \\
$\mathrm{OCN}^{-}$ & 4.61 & $\leq 3$ & $\leq 3$ & $\leq 5$ & $\leq 5-10$ & $\sim 15-30$ \\
$\mathrm{CH}_{4}{ }^{a}$ & $3.31,7.66$ & - & $\leq 0.2$ & $\sim 0.3$ & $\sim 0.3-2$ & $\sim 2-6$ \\
& & & $\leq 1.5$ & $\sim 1.9$ & $\sim 0.92-2.7$ & $\sim 1.5-3.9$ \\
\hline
\end{tabular}

See [16] for some example of sources characteristic of the different environments. Adapted from [16] and reference therein.

between $\mathrm{CO}_{2}$ and $\mathrm{CH}_{3} \mathrm{OH}$ are revealed by the complex profile of the $\mathrm{CO}_{2}$ bending mode at $15 \mu \mathrm{m}$ due to the induced lift of degeneracy of this mode [15]. The changes of the ice line profiles among different objects are important because they give insight into the evolution of the objects.

\subsection{The carbonaceous dust: VSGs, PAHs and carbonaceous grain mantles}

Interstellar carbon-rich dust may take a large variety of form from graphitic or diamond grains to amorphous (hydrogenated or not) carbon grains, carbon clusters, aromatic macromolecules, etc. Among these I will focus on three different types: (i) the Very Small Grains (VSGs), responsible for the emission 


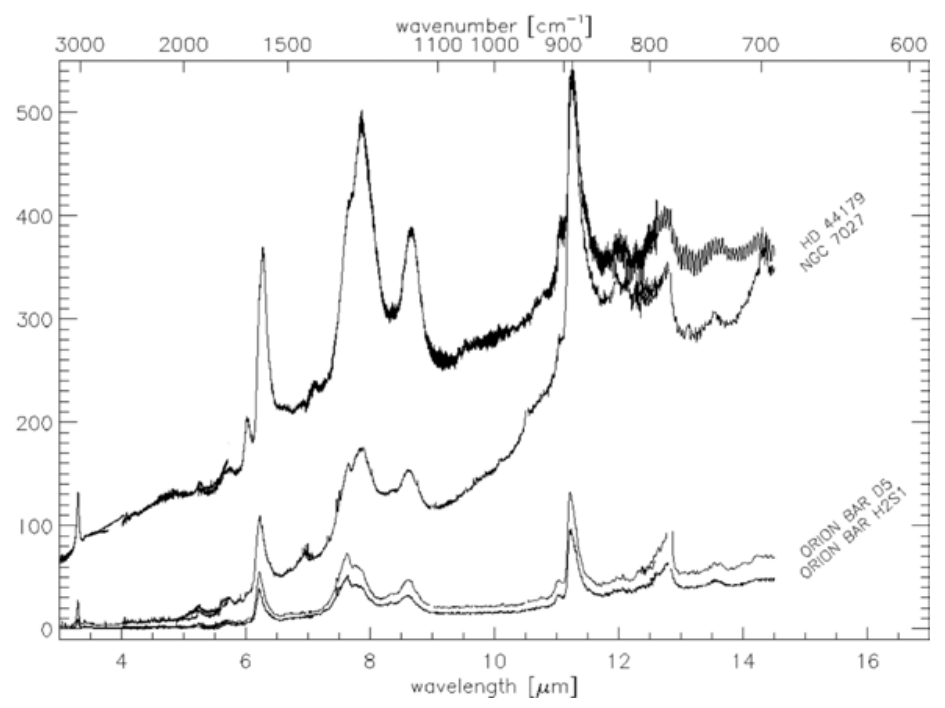

Figure 4. Mid infrared, 3-14 $\mu \mathrm{m}$, ISO-SWS spectra of the aromatic infrared bands observed in the Red Rectangle (HD 44179), NGC7027 and in two positions on the Orion ionization ridge. See Table 3 for the band assignment. From [18].

observed in the $25-60 \mu \mathrm{m}$ range by IRAS, ISO and Spitzer (Fig. 1) and whose nature is not wellconstrained, (ii) the Polycyclic Aromatic Hydrocarbons (PAHs), responsible for the Aromatic Infrared Bands (AIBs), a series of emission spectral features observed in the 3-20 $\mu \mathrm{m}$ range (Fig. 4), and (iii) the Hydrogenated Amorphous Carbon dust (HACs or a:C-H).

\section{- The VSGs}

A population of nanometer-sized grains commonly called the Very Small Grains (VSGs) is responsible for the emission observed at $\sim 25-60 \mu \mathrm{m}$ by IRAS, ISO and Spitzer (Fig. 1). Their emission is stochastic at temperatures depending on their size, their calorific capacity and the ambient UV field (several hundreds of K up to $\sim 1000 \mathrm{~K}$ ). VSGs are not clearly associated to any distinct spectral feature and, although they are usually associated to carbonaceous grains, their exact composition and structure are not well known.

\section{- The PAHs}

Polycyclic aromatic hydrocarbons (PAHs) are proposed to be the carriers of the Aromatic Infrared Bands (AIBs) observed in emission in the mid infrared $(\sim 3-20 \mu \mathrm{m})$ (see Fig. 4, Table 3). These bands are due to the IR-fluorescence of FUV-pumped PAHs molecules containing several tens of C-atoms [19]. PAHs have a strong absorption due to $\pi \rightarrow \pi^{*}$ electronic excitation near $2200 \AA$ and are thus logical contributor to the UV-bump observed in the extinction curve at $2175 \AA$ [20]. PAHs are molecules rather than dust grains, however they are considered as a grain population and their nature is closely linked to grains. They are ubiquitous and abundant, containing $\sim 10-20 \%$ of the interstellar carbon in our Galaxy [21]. The intensity of their emission is proportional to the UV radiation field, therefore PAHs are good tracers of stars forming regions, in particular in galaxies [22]. They are important for the neutral gas heating and for the ionisation balance in molecular clouds [23].

Despite 25 years of observational, experimental, and theoretical studies, no individual PAH molecule has been identified yet. The IR features do not help much as they are sensitive to the chemical bonds 
Table 3. List of the observed AIBs associated to the vibrational modes of PAHs.

\begin{tabular}{ll}
\hline observed bands & spectroscopic mode \\
\hline $3.3 \mu \mathrm{m}$ & C-H stretch \\
$6.2 \mu \mathrm{m}$ & C-C stretch \\
$7.7 \mu \mathrm{m}$ & C-C stretch \\
$8.6 \mu \mathrm{m}$ & C-H bend in-plane \\
$11.3 \mu \mathrm{m}$ & C-H bend out-of-plane (solo) \\
$12.7 \mu \mathrm{m}$ & C-H bend out-of-plane (duo-trio) \\
$13.6 \mu \mathrm{m}$ & C-H bend out-of-plane (duo-trio-quatro) \\
$16.4 \mu \mathrm{m}$ & out-of-plane skeletal vibrations \\
$17.4 \mu \mathrm{m}$ & out-of-plane skeletal vibrations \\
\hline
\end{tabular}

and are not specific of a distinct molecule. Although electronic transitions are more characteristic of the overall molecular structure, no clear identification of any specific PAHs was made except maybe for the tentative identifications of naphthalene and anthracene cations from two DIBs (Diffuse Interstellar Bands) [24, 25]. Low-lying ro-vibrational transitions due to the deformation of the carbon skeleton may help to identify PAHs but their emission is weak and their detection is a challenging task for the Herschel mission [26].

Interstellar PAHs may be neutral, or ionised depending on the local radiation field and the electron density. They contain from a few tens to several hundreds of carbon atoms and may be dehydrogenated or super-hydrogenated depending on the environment. The profile, peak position and substructure of the AIBs give information on the size, ionisation state, composition of the PAHs. In particular, the degree of ionisation is deduced from the $7.7 / 11.3 \mu \mathrm{m}$ band ratios, the $\mathrm{C}-\mathrm{H}$ bending mode at $11.3 \mu \mathrm{m}$ being more intense relative to the $\mathrm{C}-\mathrm{C}$ stretching mode at $7.7 \mu \mathrm{m}$ in neutral PAHs compare to cations.

\section{- Hydrogenated amorphous carbon}

The interstellar band at $3.4 \mu \mathrm{m}$ observed toward diffuse Galactic line of sights was long ago attributed to methyl $\left(-\mathrm{CH}_{3}\right)$ and methylene $\left(-\mathrm{CH}_{2}\right)$ groups in amorphous carbonaceous dust (the Hydrogenated Amorphous Carbon: HAC, also designated by a:C-H) [27]. This band is associated with two other absorption features at 6.85 and $7.25 \mu \mathrm{m}$ attributed to the bending modes of these methyl and methylene groups [28]. This grain population, which represents $\sim 5$ to $30 \%$ of the carbon cosmic abundance, is also widespread, as revealed by its observation in external galaxies [28]. The band at $3.4 \mu \mathrm{m}$ is also observed in emission, together with the AIBs, in some proto-planetary nebulae in which it was proposed to be due to PAHs containing methyl groups [29]. The changes in the shape and relative intensity of these bands in the different environments where they are observed reflect the dependence of the carrier composition on the ambient conditions. Comparison of observational and laboratory spectra shows that hydrocarbons in the diffuse ISM (DISM) contain more methyl groups than in dark clouds whereas methylene groups are always present [30].

Hydrogenated amorphous carbon, a:C-H, may be in the form of a refractory mantle surrounding silicates grains or may constitute a distinct grain population, it is not settled yet. Polarisation observations show that the $3.4 \mu \mathrm{m}$ interstellar band is not polarised whereas the $9.7 \mu \mathrm{m}$ is polarised, suggesting that the carriers of this band do not reside on the same grains but exist as a separate grain population [31]. On the other hand, these features are well reproduced experimentally via UV photolysis of ices resembling interstellar ices [32], suggesting that a:C-H may be in the form of mantles around silicate grains. 

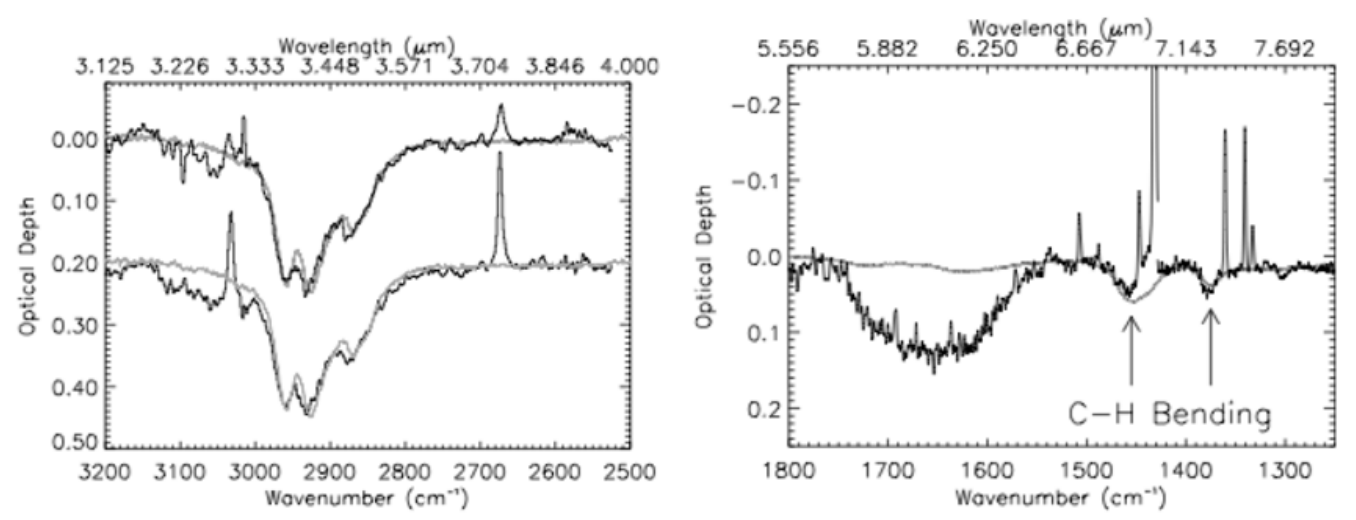

Figure 5. Optical depth of the Galactic Center IRS7 line of sight (black curves) showing the C-H stretch region (left) and C-H bend region (right). The grey curves are the optical depth of photo-produced hydrogenated amorphous carbon. From [17].

\section{DUST FORMATION}

Cosmic dust forms principally in the cold gas outflowing from evolved stars (Asymptotic Giant Branch (AGB) stars, red giant branch stars, cool supergiants) and in the ejecta resulting from supernova (SN) explosions. The contribution of SN explosions to the overall budget of dust formation is difficult to estimate (see the review from Kozasa et al. [33]). However it is secure to consider evolved stars, and among them AGB stars, as the main source of cosmic dust. Around cold stars, dust is formed in dense cool shells created by stellar pulsations or large-scale convective motions which propagate gas above the stellar surface (Fig. 6, [34]). These dust grains are then accelerated away from the star by radiation pressure and eventually feed the ISM.

In circumstellar shells, dust forms from the matter already present when the stars formed and from the elements produced by the stars itself. Consequently the newly produced dust composition changes as the star evolves and in particular, when the carbon to oxygen ratio changes. When $\mathrm{C} / \mathrm{O} \leq 1$ (Mstars), all carbon is bound in the $\mathrm{CO}$ molecule and the oxygen in excess participates to oxygen-rich dust production. When $\mathrm{C} / \mathrm{O} \geq 1$ (C-stars) carbon is in excess while all the oxygen is bound in $\mathrm{CO}$ and hydrocarbons molecules and carbon grains are formed. When $\mathrm{C} / \mathrm{O} \approx 1$ (S-stars), only small amount of dust may be formed since there is not much material available to produced it. In addition, dynamical effects are important in circumstellar shells and outflows and are thus expected to play a role in the dust formation process.

\subsection{Nucleation and growth of oxygen-rich dust}

The formation of dust takes place in two steps. During the first one, the nucleation phase, the gas phase condensates to form a stable nucleus (see the contribution from P. Parneix, this volume, on the theory of nucleation). The second one is the growth of the grain via the accretion onto the nucleus of the ambient gas, atoms or molecules. Grain nucleation and growth have been largely studied theoretically ([35, 36] and reference therein). Most of these studies consider thermodynamical equilibrium and only recently take into account the effects of dynamics ([37] and reference therein). However, because it is difficult to produce a gas with a controlled composition of refractory elements and to condense it at high temperature [38], few experimental studies of dust formation exist.

Recent experiments performed in conditions relevant to the stellar/solar dust formation, i.e., high gas temperature, high condensation temperature and low pressure, have given some answers to the cosmic dust formation process [39, 40]. Experiments from [39], performed at equilibrium, are interesting as 


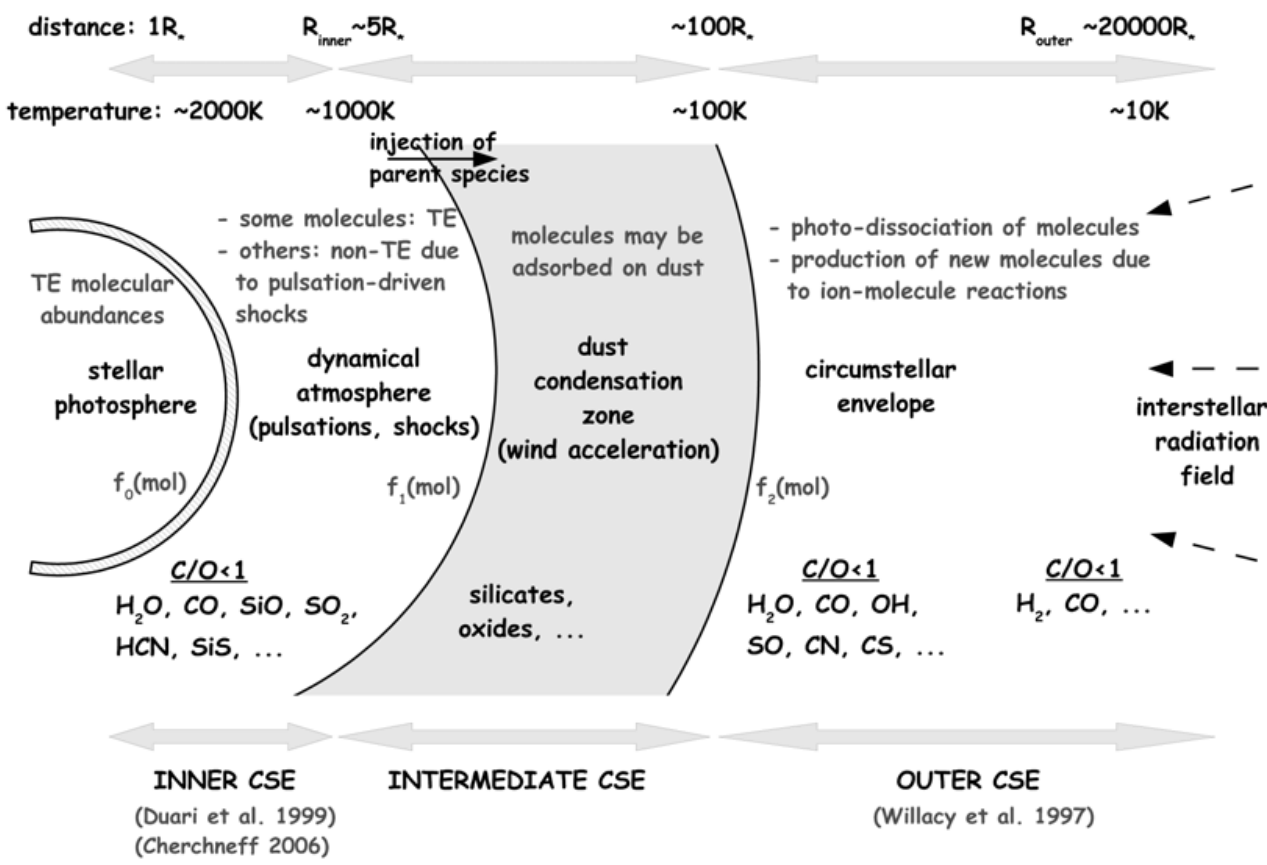

Figure 6. Schematic overview (not to scale) of dust formation around oxygen-rich evolved stars. Taken from [34].

they allowed the authors to study the dust nucleation since they observed grains which condensed in the gas phase and not on a substrate. They find that nucleation and growth occur simultaneously and find some hints of forsterite grains with $\mathrm{Al}_{2} \mathrm{O}_{3}$ core. These experiments show that, as expected, the composition of the ambient gas influence the composition of the newly made dust. Starting from a gas composition close to the solar nebula, forsterite, enstatite, corundum, spinel, melilite are produced principally: all material observed in cosmic dust. Crystals are formed directly from the gas whereas no amorphous material is produced. On the other side, different experiments show that amorphous $\mathrm{Mg}$-rich silicates may be produced by deposition onto an $\mathrm{Al}_{2} \mathrm{O}_{3}$ substrate at temperature that varies with the gas kinematic composition, and pressure [40]. $\mathrm{Al}_{2} \mathrm{O}_{3}$ is observed in evolved stars where the dust forms and is proposed to be the nucleation seed for the silicate growth. Around evolved stars, both crystalline and amorphous silicates are observed together with various oxides. Although the crystalline silicates composition is relatively well known, the one of the amorphous component is poorly constrained. These experiments thus raise many questions that have yet to be answered: is the crystalline dust amorphized in the circumstellar envelope? Are the physical, chemical and/or dynamical conditions different from the experiments? What is the composition of the amorphous silicate component?

\subsection{Carbon-rich dust formation}

Carbon dust may take a large variety of forms: large amorphous carbonaceous or graphitic grains, very small grains of a few nm, large molecules such as PAHs. Studying its formation is thus a complex task. However, as for the O-rich dust, evolved stars are major contributors to the carbon rich cosmic dust. In these environments, the formation of carbon nanoparticles and PAHs is thought to proceed via the nucleation of small PAHs followed either by reaction with acetylene $\left(\mathrm{C}_{2} \mathrm{H}_{2}\right)$ or the dimerization of PAHs such as pyrene to form larger PAHs and nanograins [41-43]. These scenarios are inspired by soot formation and combustion studies. However, they are not fully understood. In particular, recent experimental studies have shown than the dimerization of pyrene is very inefficient [44]. The readers 


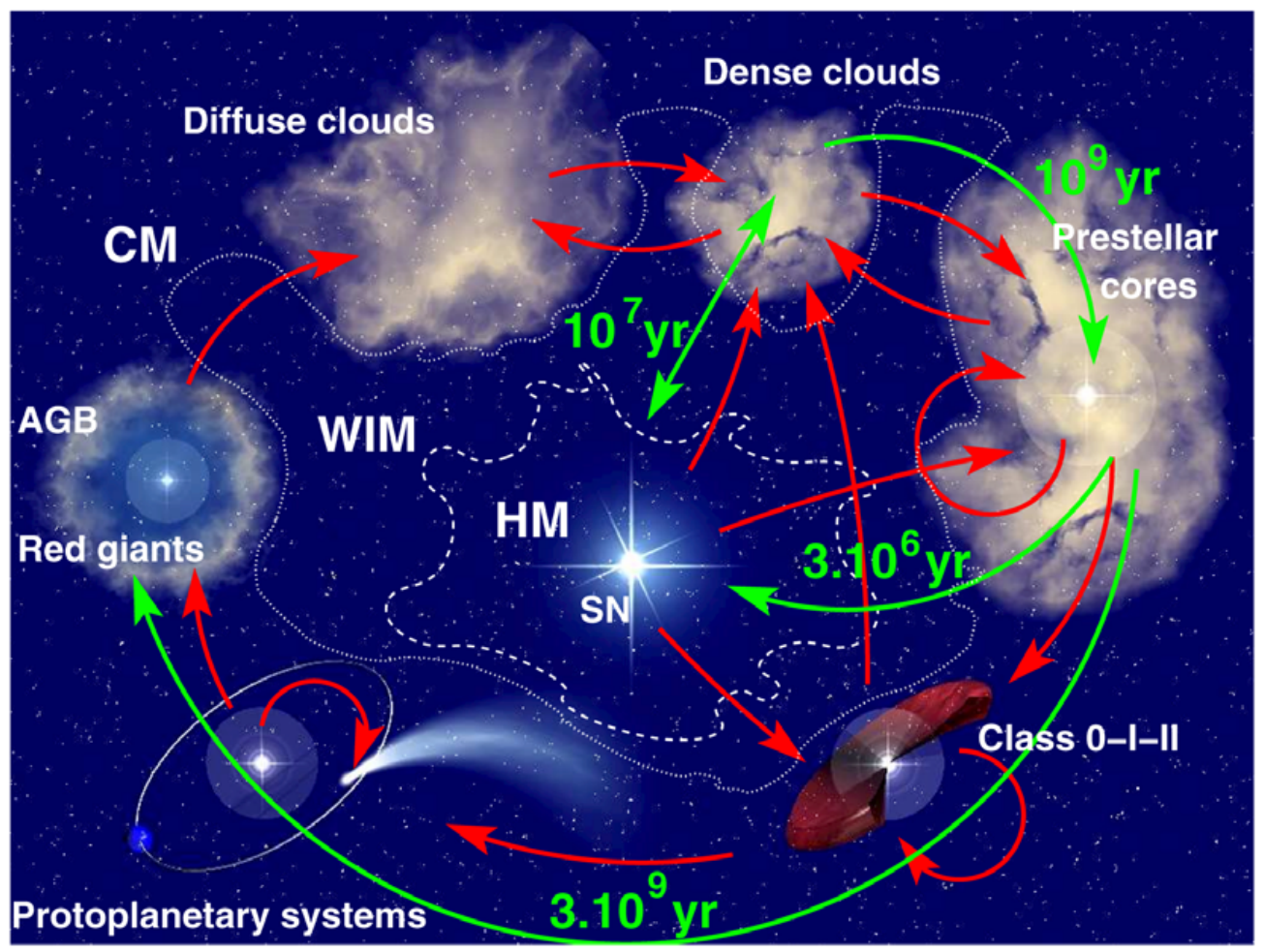

Figure 7. Interstellar medium cycle showing the interaction between the different ISM phases: the warm intercloud medium (WIM), the hot medium (HM) and the cold medium (CM). Taken from [32].

are referred to the contribution from P. Desgroux in this volume for a review on this subject. As for the oxygen-rich dust formation, the formation of the nucleation seed, the small PAHs in this case, is crucial and not well understood. Models of AGB winds and outflows that incorporate results from terrestrial soot studies are not able to produce efficiently PAHs and therefore fail to explain the observed abundance of PAHs and carbonaceous grains [45, 46].

\section{DUST EVOLUTION AND DESTRUCTION}

During its lifetime, cosmic dust is submitted to many processes as it goes from diffuse to dense clouds or during the collapse of dense molecular clouds. These processes may destroy the grains, violently, by shattering, or more gently, by sputtering. Dust evolution models estimate the destruction timescale of grains in the ISM to be $\sim 6 \times 10^{8}$ and $4 \times 10^{8}$ years for graphite and silicates, respectively [47]. From these numbers, some authors conclude that up to $90 \%$ of the dust grains in the ISM is not formed around stars but in the ISM itself and that the regrowth of dust in the ISM is required to explain the observed depletion $[48,49]$. As it seems very unlikely that dust nucleation could take place in interstellar clouds, the proposed mechanism for grain growth in the ISM is via accretion of atoms and molecules from the gas phase onto pre-existing surfaces [20]. However further modelling and experiments are needed to test the plausibility of this scenario and the efficiency of such a mechanism and its ability to form silicates and carbonaceous dust at low temperature and pressure much lower than those encounter in circumstellar envelopes.

In any case this illustrates the efficiency of the coupling between the dust and the environment to deeply modify the dust nature. Indeed, processes that do not destroy the grains modify their physical and 
chemical properties. Grain coagulation in cold clouds increases the grains size [50]. Shock waves or supernovae explosions replenish the ISM in smaller grains as it shatters big grains preferentially. They also induce an important irradiation of the grains by the ionised gas accelerated that can destroy the structure of the grains and change their composition [51]. In cold dense clouds atoms and molecules from the gas phase condense onto the grains to form an icy mantle. Grains and their mantles provide surfaces on which surface and solid-state chemistry may take place. They are submitted to UV photolysis and irradiation by cosmic rays that induce a rich chemistry essential to explain the formation of large complex molecules and refractory carbonaceous mantles. In hotter environments grains participate to the enrichment of the gas phase via thermal or explosive desorption of the icy mantles or via photo-evaporation of carbonaceous nanograins and PAHs that release small hydrocarbons in photodissociation regions (PDR).

\subsection{Icy mantles: Location of a rich chemistry}

Interstellar icy mantles may evolve via surface reactions, UV photolysis, cosmic rays irradiation or thermal cycling. These processes change the composition, the structure of the ices but also the composition of the gas-phase since molecules from the ices desorb from the mantles to enrich the ambient gas. The relative importance of these processes, which is still a matter of debate, varies according to the astronomical environment.

\section{- UV photolysis and cosmic rays irradiation: Formation of complex molecules}

In interstellar environments, UV photons may come from the stellar radiation, the UV background field, and from the effect of cosmic rays excitation of $\mathrm{H}_{2}$. This last source of UV photons dominates in the inner part of dense clouds where the background UV field is attenuated, ensuring that a minimum UV field is always present in all environments. Cosmic rays are ubiquitous and penetrate the densest regions in which they are only weakly attenuated. UV photons and cosmic rays interact differently with the ice. First, the penetration depth of the UV photons and cosmic rays are very different: a few tens of $\mu \mathrm{m}$ for the UV photons whereas cosmic rays may go through the grains [56]. The second difference is the absence of threshold energy for cosmic rays whereas UV photons energy is smaller than $13.6 \mathrm{eV}$, the ionisation potential of $\mathrm{H}_{2}$. There is a longstanding debate on the relative importance of UV photolysis relative to cosmic rays bombardment and it is difficult to observe evidence favouring one or the other process as observing the result of the reaction do not usually allows to discriminate between the distinct possible formation pathways. However one may estimate that the energy deposited on the grains by UV photons induced by the cosmic rays is about one order of magnitude greater than the energy deposited directly by the cosmic rays irradiation [52].

Laboratory work on UV photolysis and cosmic rays irradiation of interstellar ice analogs have for a long time shown their efficiency to produce the major observed ice molecules such as $\mathrm{CO}_{2}$ or $\mathrm{CH}_{3} \mathrm{OH}$ $[53,54]$. Such processes are also proposed to form more complex molecules, not detected in interstellar ices but observed in the gas phase, and which are thought to be formed within the ice mantles and released into the gas phase. Several reasons prevent their detection in interstellar ice spectra: a low abundance in the ice, weak lines and/or the spectral blending of the lines with abundant species in the ice. Large complex molecules such as methyl formate, ethanol, dimethyl-ether, and many others are commonly observed in the gas phase of hot cores in which the dust reaches temperature greater than $100 \mathrm{~K}$. Chemistry models of formation of these molecules in the gas phase are unable to account for their observed abundance and most models now consider gas-grain chemistry in addition to gas-phase chemistry [55].

Many experiments are now dedicated to the production of complex molecules in the ices. Electronic irradiation were used to mimic the effect of $\mathrm{MeV}$ cosmic-rays irradiation of ices to produce the three isomers: acetic acid $\left(\mathrm{CH}_{3} \mathrm{COOH}\right)$, methyl formate $\left(\mathrm{HCOOCH}_{3}\right)$ and glycolaldehyde $\left(\mathrm{HCOCH}_{2} \mathrm{OH}\right)$ $[57,58]$. They are also produced by UV photolysis of ices together with acetaldehyde $\left(\mathrm{CH}_{3} \mathrm{CHO}\right)$, ethanol $\left(\mathrm{CH}_{3} \mathrm{CH}_{2} \mathrm{OH}\right)$, dimethylether $\left(\mathrm{CH}_{3} \mathrm{OCH}_{3}\right)$ illustrating that the overall chemistry induced by $\mathrm{UV}$ 
photolysis and ion irradiation is similar [59]. Many other complex molecules have been experimentally studied such as cyclopropenone $\left(\mathrm{c}-\mathrm{C}_{3} \mathrm{H}_{2} \mathrm{O}\right)$ and propynal (HCCCHO) [60], carbamic acid $\left(\mathrm{NH}_{2} \mathrm{COOH}\right)$ and ammonium carbamate $\left(\mathrm{NH}_{2} \mathrm{COO}^{-} \mathrm{NH}_{4}^{+}\right)$[61].

\section{- Surface reactions}

In surface reactions, atomic hydrogen and oxygen play a major role. The atomic hydrogen abundance in clouds is governed by the destruction of $\mathrm{H}_{2}$ by the cosmic rays and is independent of the cloud density $\left(\mathrm{n}_{H} \sim 1 \mathrm{~cm}^{-3}\right)$ whereas the oxygen atomic density depends on the cloud density and increases with the $\mathrm{H}_{2}$ density $\left(\mathrm{O} / \mathrm{H}_{2} \sim 10^{-4}\right)$. In dark clouds, the first monolayer of $\mathrm{H}_{2} \mathrm{O}$ ice forms on bare silicate and carbonaceous grains, and once this monolayer is formed new molecules form on $\mathrm{H}_{2} \mathrm{O}$ ice surface. When the ice composition has become more complex, surface reactions occur on surfaces composed of several molecules. The ice temperature is also an important parameter since when the temperature is too high the residence time of the incident atoms gets shorter.

Water ice formation has been studied via $\mathrm{H}$ and $\mathrm{O}$ atoms and $\mathrm{O}_{2}$ bombardment of various substrates: $\mathrm{O}_{2}$, crystalline and amorphous $\mathrm{H}_{2} \mathrm{O}$ ices. Interaction of $\mathrm{H}$ atom with $\mathrm{O}_{2}$ surface at various temperatures produces $\mathrm{H}_{2} \mathrm{O}_{2}$ and $\mathrm{H}_{2} \mathrm{O}$ [62-64]. $\mathrm{H}$ and $\mathrm{O}$ atoms bombardment of amorphous $\mathrm{H}_{2} \mathrm{O}$ surface forms efficiently $\mathrm{H}_{2} \mathrm{O}$ at temperature lower than $20 \mathrm{~K}$ via $\mathrm{H}+\mathrm{O}$ reaction [65]. Numerous experimental studies have focussed on the formation of larger molecules such as $\mathrm{CO}_{2}, \mathrm{H}_{2} \mathrm{CO}, \mathrm{CH}_{3} \mathrm{OH}$ and their deuterated isotopologues (see the recent review by Wanatabe \& Kouchi, [66] and reference therein). $\mathrm{H}$ bombardment of pure carbon dioxide $\left(\mathrm{CO}_{2}\right)$, formic acid $(\mathrm{HCOOH})$ and acetaldehyde $\left(\mathrm{CH}_{3} \mathrm{CHO}\right)$ ices have shown that hydrogenation of $\mathrm{CO}_{2}$ and $\mathrm{HCOOH}$ is negligible in interstellar chemistry networks whereas hydrogenation of $\mathrm{CH}_{3} \mathrm{CHO}$ leads to the formation of ethanol $\left(\mathrm{C}_{2} \mathrm{H}_{5} \mathrm{OH}\right)$, methane $\left(\mathrm{CH}_{4}\right)$, formaldehyde $\left(\mathrm{H}_{2} \mathrm{CO}\right)$ and methanol $\left(\mathrm{CH}_{3} \mathrm{OH}\right)$ and may explain the observed abundance of ethanol [67].

As for the role of UV photolysis versus cosmic rays irradiation, there is a debate on the importance of surface reactions versus energetically assisted processes [68], a good example illustrating this being methanol. Laboratory experiments have shown that the gas phase ion-neutral reactions are insufficient to produce methanol with the observed abundance [69]. Methanol, which is observed in interstellar ices, must thus be formed, at least partly, in the ices. However, to date it is not settled wether it forms on the grains via UV photolysis, or surface reactions. Experiments show that $\mathrm{CH}_{3} \mathrm{OH}$ is formed via $\mathrm{CO}$ hydrogenation $[70,71]$ at temperature smaller than $15 \mathrm{~K}$ and at high $\mathrm{H}$ fluence $\left(\geq 10^{14}\right.$ atoms.cm $\left.{ }^{-2} \cdot \mathrm{s}^{-1}\right)$, compatible with the interstellar conditions. However, because the penetration depth of the $\mathrm{H}$ atom at $12 \mathrm{~K}$ is estimated to $\sim 4$ monolayers of solid $\mathrm{CO}$, this process can hardly account for the very high methanol abundance observed in some protostellar objects: up to $25 \%$ of the water ice [72] which is equivalent to $\sim 25$ monolayers of methanol [73]. While it is proposed that the formation of methanol occurs simultaneously with the freeze-out and building of the $\mathrm{CO}$ layer, another explanation could simply be that $\mathrm{CH}_{3} \mathrm{OH}$ is also formed via the UV photolysis of the ices that contains $\mathrm{H}_{2} \mathrm{O}$ and $\mathrm{CO}$ in protostellar objects.

In order to understand the reaction route leading to the produced molecules, all experiments of surface reactions use a pure ice surface ( $\mathrm{CO}$ in the case of the $\mathrm{CH}_{3} \mathrm{OH}$ formation). However it is known that interstellar ices are composed of many molecules that are intimately mixed and a mono-component surface is unlikely to exist. Multi-component surface may change the efficiency of the reactions but may also change the reaction route producing the molecules. Further experiments performed on more realistic ice surfaces made of several molecules are clearly needed.

\section{- Thermal annealing}

Chemical reactions also occur during the annealing of the grains in protostellar objects. Their efficiency depends on the mobility of the ice species, and hence on the ice temperature, but also on the presence of radical in the ice. The increased mobility of the ice species will favour the formation of complex molecules prior to their desorption. As an example, thermal processing of $\mathrm{H}_{2} \mathrm{O}: \mathrm{NH}_{3}: \mathrm{H}_{2} \mathrm{CO}=10$ : $5: 0.3$ ices shows that ammonia and formaldehyde start to react at $\sim 80 \mathrm{~K}$ to form an organic residue 
containing aminomethanol $\left(\mathrm{NH}_{2} \mathrm{CH}_{2} \mathrm{OH}\right)$ [74]. Annealing of $\mathrm{UV}$ photolyzed ices can form a refractory organic mantle containing oxygen and nitrogen molecules of prebiotic interest [75].

- Desorption: Enrichment of the interstellar gas

An obvious desorption mechanism of the ice mantle is thermal desorption which was experimentally studied by different groups [76, 77]. Although it is an important mechanism in protostellar object where the grains reach high temperature, in cold interstellar clouds where ices are present, the temperature is too low. However, depletion of the gas phase and molecules on the dust surface being very efficient, models predict that the ISM should be gas-free in $\sim 10^{5}$ years [78]. As it is obviously not the case, some mechanisms must released into the gas-phase part of the matter contained in the grains. Refractory elements return to the gas-phase when grains are submitted to sputtering and shattering in shocks, in turbulent clouds and these processes also affect the ice mantle. Other mechanisms are UV photodesorption and explosive desorption induced by cosmic rays. UV photodesorption rate have been measured experimentally for the major ice species : $\mathrm{H}_{2} \mathrm{O}, \mathrm{CO}, \mathrm{CO}_{2}$, and $\mathrm{CH}_{3} \mathrm{OH}[59,79,80]$. Explosive desorption may occur when the energy, deposited by UV photons and/or cosmic rays and stored into the radicals in the ice, is released during transient heating of the grains that may be due to grain-grain collisions [81]. The preferential desorption of small molecules during this process could lead to the ice segregation with an inner layer containing large non-desorbed molecules and the outer layers containing the volatile small molecules that have re-condensed onto the ice surface [52].

\subsection{Evolution of carbon rich dust}

The study of the evolution of carbon dust may be the opportunity to investigate the possible link between the various forms it can take. PAHs, VSGs, large carbon grains may be considered as distinct populations of cosmic dust that evolve independently from each other and we can study their physical and chemical processing separately. However the processing of these species may reveal connections between them, connections that are suggested by the observations. In the following, some elements of the carbon rich dust evolution are presented. However, for an exhaustive review on PAHs and carbon rich dust the reader is invited to refer to the proceedings of the Symposium "PAHs and the Universe" held in Toulouse in May 2010 [82].

\section{- Photochemistry of PAHs}

Photodissociation regions are characterised by a strong UV radiation field that may change deeply the nature of the carbon dust. Interferometric observations of the Horsehead nebula show that small hydrocarbons molecules $\left(\mathrm{CCH}, \mathrm{c}-\mathrm{C}_{3} \mathrm{H}_{2}, \mathrm{C}_{4} \mathrm{H}\right)$ are present in the $\mathrm{UV}$-irradiated gas where intense PAHs mid-infrared bands are observed [83]. This suggests that these molecules could be produced by UV irradiation of PAHs and small carbon grains. Experiments show that $\mathrm{C}_{2} \mathrm{H}_{2}$ is the major channel of fragmentation for small PAHs $\left(\mathrm{N}_{C} \leq 14\right)$ [84] whereas for larger species (coronene or di-coronene) the destruction of the carbon skeleton occurs only after complete dehydrogenation by the successive loss of all $\mathrm{H}$ atoms [85].

The photochemistry of PAHs aggregates and metal-PAHs clusters have also been experimentally study. Under visible irradiation from a Xenon lamp, metal-PAHs clusters photo-dissociate into the PAH molecule and the metal atom, the building blocks of the clusters: $\mathrm{Fe}\left(\mathrm{C}_{24} \mathrm{H}_{12}\right)_{2}^{+}$clusters first lose a coronene molecule and then the iron atom [86]. Similarly, experiments on coronene clusters $\left(\left(\mathrm{C}_{24} \mathrm{H}_{12}\right)_{n}\right.$ with $\mathrm{n} \leq 13$ ) submitted to UV laser irradiation show that the clusters lose coronene molecules [87, 88].

\section{- VSGs and PAHs production in the ISM}

While PAHs are proposed to feed the gas-phase with small molecules resulting from their photoevaporation, it is proposed that PAHs in photo-dissociation regions (PDRs) are themselves produced in situ by the destruction of nanometric carbon grains caused by UV radiation and/or shock waves 


\section{EPJ Web of Conferences}

$[89,90]$. These grains are characterised by a strong rising mid infrared continuum and broad bands with a low contrast against the continuum, as shown by spectral mapping observations of the reflexion nebula Ced201[89]. A similar spectrum results from a multi-spectral analysis of Spitzer photometric maps of different objects and is attributed by the authors to small carbon grains [90]. Using this method, observations of a large number of astronomical objects are well-modelled with three or four synthetic spectra which may be associated to measured or calculated spectra of, respectively, large ionized PAHs, singly ionized PAHs, neutral PAHs and carbonaceous nano-grains [91, 92]. These spectra have separated spatial distributions, the nanograins being the more distant from the star, the neutral PAHs closer and the singly and multiply ionized PAHs the nearest. These carbonaceous nanograins may be considered as the smallest VSGs $(\sim 1 \mathrm{~nm})$ but their nature is unknown. They could be composed of amorphous hydrogenated carbon [89] or PAHs clusters [93]. If PAHs clusters are dissociated in PDRs, they must be formed somewhere in protected regions of the ISM such as cold dense clouds. Models show that in interstellar conditions, most collisions between PAHs molecules lead to PAH cluster growth [94]. Experiments on coronene clusters show that they photo-dissociate into coronene molecules [87] which is compatible with the above scenario.

- Classification of the AIBs: Toward a scenario for the carbon-rich dust evolution?

The spectral signatures of the AIBs (Aromatic Infrared Bands) present some variations in terms of band position and relative intensity. Three classes (A, B and C) have been defined based on the changes of the bands at 6.2 and $7-9 \mu \mathrm{m}$, the bands at 3.3 and $11.3 \mu \mathrm{m}$ being mostly unmodified [95]. The $6.2 \mu \mathrm{m}$ band shifts progressively to $6.3 \mu \mathrm{m}$ from class A to class C. The 7-9 $\mu \mathrm{m}$ complex is composed of two bands at $7.7 \mu \mathrm{m}$ (with a shoulder at $7.8 \mu \mathrm{m}$ ) and $8.6 \mu \mathrm{m}$ in class A spectra. These bands shift toward longer wavelengths in class B spectra (the relative intensity of the 7.7 and $7.8 \mu \mathrm{m}$ is inverted) whereas only one broad band at $\sim 8.2 \mu \mathrm{m}$ is observed in class $\mathrm{C}$ spectra. Class A spectra are observed in HII regions, reflexion nebulae, extragalactic sources, post-AGB stars and planetary nebulae, class $\mathrm{B}$ spectra in isolated Herbig Ae/Ba stars, post-AGBs stars and planetary nebulae and class C spectra only in post-AGBs stars and young planetary nebulae. PANHs, i.e., polycyclic aromatic molecules with some carbon atoms substituted with nitrogen atoms, have been proposed to explain these variations [96]. However, while calculations show that the $6.2 \mu \mathrm{m}$ is shifted toward long wavelengths in PANHs, the other bands are not well reproduced, as shown from spectroscopic measurements of gas-phase PANHs [97].

An alternative explanation, based on experimental studies of carbon dust analogs and on the fact that class $\mathrm{C}$ spectra present an emission band at $3.4 \mu \mathrm{m}$, establishes an evolutionary link between aliphatic and aromatic dust by proposing that class $\mathrm{C}$ spectra are characteristic of a mixture of aromatic and aliphatic carbon-rich dust $[98,99]$. Infrared spectrum of soots containing different amount of aliphatic versus aromatic carbons shows that when the aliphatic/aromatic carbon ratio increases the changes in the $6.2 \mu \mathrm{m}$ band and in the 7-9 $\mu \mathrm{m}$ complex are in agreement with a change from class A to class $\mathrm{C}$ spectrum. In the $3 \mu \mathrm{m}$ region, the $3.3 \mu \mathrm{m}$ band of PAHs disappears while a band at $3.4 \mu \mathrm{m}$ characteristic of aliphatic carbon appears and the 11-13 $\mu \mathrm{m}$ region does not change significantly [99]. The authors proposed that the band at $6.2 \mu \mathrm{m}$ is a tracer of the evolution of the carbon matter via the aliphatic/aromatic ratio. They proposed that both around post-AGB stars and in PDRs, when the UV stellar field increases, the aliphatic-dominated grains are processed and transformed into an aromatic dominated carbon component while their mid-infrared spectrum becomes similar to class B and then to the widespread class A spectrum. Indeed a connection seems to exist between the VSGs spectrum resulting from the multi-spectral analysis of PDRs map [91, 93] and class B and C spectra. Other models of carbon-rich dust implying an evolution from aliphatic to aromatic dust have been proposed [100]. Based on the multi-spectral analysis from [93], it explains the spectral changes of the $7.7 \mu \mathrm{m}$ in evolved stars by the transformation of aliphatic dust into large aromatic dust under harsh UV irradiation. 


\subsection{Evolution of silicate dust}

\section{- Crystalline to amorphous transformation in the ISM}

The silicate dust formed around evolved stars and injected into the ISM is partly crystalline and partly amorphous, in proportion that are not known. However it is known from ISO and Spitzer observations that the grains in the ISM are amorphous to more than 98\% [101, 102]. Consequently, processes must exist in the ISM that destroy the crystalline silicates and/or amorphize them. To modify the nature of the refractory oxygen rich dust, high energetic processes such as cosmic rays bombardment or high velocity shocks are required, two processes that occur in the ISM. Cosmic rays bombardment efficiently destroy the structure of crystalline material as it induces the displacement of the atoms within the lattice. It is the most efficient when the incident ion is implanted into the grains. Energetic shocks resulting from supernovae explosions mimic the ions bombardment as they accelerate the grains to high velocities while the gas ionised by the shock stay still. A $700 \mathrm{~km} / \mathrm{s}$ shock is similar to a $4 \mathrm{keV} \mathrm{He}{ }^{+}$irradiation of the grains. Experiments of ion irradiation of dust analogs have proven that these processes may fully amorphized all the crystalline grains in conditions relevant to interstellar medium [51, 103-105]. Such an irradiation also changes the composition of the silicate from olivine-type silicates $\left(\mathrm{Mg}_{2 \mathrm{x}} \mathrm{Fe}_{2-2 \mathrm{x}} \mathrm{SiO}_{4}\right)$ toward pyroxene-type silicates $\left(\mathrm{Mg}_{\mathrm{x}} \mathrm{Fe}_{1-\mathrm{x}} \mathrm{SiO}_{3}\right)$ by the preferential loss of $\mathrm{Mg}$ [51].

\section{- Shattering and coagulation in the ISM}

The size distribution of interstellar grains is derived form the observations of the interstellar extinction curve. It results from a balance between sputtering, shattering, coagulation that can take place in the different ISM phases and during the exchange between these phases [106]. When the relative gas-grain velocity exceed a few $\mathrm{km} \cdot \mathrm{s}^{-1}$ the larger grains are shattered whereas at lower velocity the small grains coagulate [47]. Furthermore the turbulent and magnetised properties of the ISM influence the relative gas-grain velocity and consequently the above mentioned processes [107]. Shattering is most efficient in the warm ionised medium (WIM) and governs the maximum interstellar grain size ( $\leq 1 \mu \mathrm{m}$, [107]). Coagulation mainly takes place in molecular clouds and dark clouds and coagulation of small grains is favoured since they have lower velocity than larger grains [107]. Observations by the balloon mission PRONAOS of several regions across a filament in the Taurus clouds reveal an increase of the brightness at the centre of the molecular filament, suggesting that the dust properties change inside the filaments. These observations, together with the decrease of the dust temperature inside the filament (from $\sim 15$ to $\sim 12.5 \mathrm{~K}$ ), are well reproduced by taking into account grain coagulation resulting the formation of fluffy aggregates composed of big silicate grains and small carbonaceous grains, the VSG [50].

\section{- Silicates in proto-planetary objects}

Interstellar dust is incorporated into protostellar objects when molecular clouds collapse. The newly born pre-main sequence star is then surrounded by a proto-planetary disk that contains the remaining interstellar dust. Proto-planetary disks are important objects where planets, comets and asteroids form. The large width of the $9.7 \mu \mathrm{m}$ band in proto-planetary disks reflects the growth of the silicate dust from small grains with sizes characteristic of interstellar dust $(\sim 0.1 \mu \mathrm{m})$ up to several microns [108]. Grains larger than a few $\mu \mathrm{m}$ are not observable because the bands at 9.7 and $18 \mu \mathrm{m}$ are more and more distorted as the grain size increases and finally vanish.

Proto-planetary disks represent good objects for the analogy with the solar nebula and for the study of the formation of our own Solar System (see the contributions from C. Engrand and L. Rmusat, this volume). The ISO observations of the comet Hale-Bopp and the proto-planetary disk surrounding the Herbig star HD100546 reveal striking and puzzling similarities of the dust properties in these two environments [109]. Although it confirms the pertinence of the analogy mentioned above, it also had strong implications on models of the evolution of the pre-solar nebula. The ISO spectra show that a large part of the dust in these two objects, and in most proto-planetary disks too, is crystalline and composed mainly of forsterite $\left(\mathrm{Mg}_{2} \mathrm{SiO}_{4}\right)$ [109]. However, it was thought that comets form in 
the outer region of the Solar Nebula from interstellar dust. Cometary dust was thus believed to be representative of interstellar dust. The dust in the ISM is amorphous and the presence of forsterite in proto-planetary disks and in comets implies that the interstellar dust have been processed to high temperature to crystallise it. This may happen if the dust gets closer to the central star where it is annealed or completely destroyed and then re-condensed [110, 111]. However, some mechanisms must exist, such as radial mixing of the nebulae and disks, to transport the dust crystallised near the star toward the outer cold region where it is observed and where comets form [112]. For a detailed review of the dust evolution in proto-planetary nebulae, the reader should refer to the recent review by Henning \& Meeus [113].

\section{CONCLUSION: SOME OPEN QUESTIONS}

Our knowledge of the nature and evolution of interstellar dust has made tremendous progress these last few decades, mostly thanks to infrared spectrometer and spectro-imager onboard space-born missions and thanks to the experimental effort undertaken in order to identify the cosmic dust and to simulate its evolution in various astrophysical environments. Nevertheless, many questions remains unanswered. The nature of amorphous dust, of silicate-type or carbon-rich type is difficult to investigate because of the lack of well-defined spectral feature and the non uniqueness of the possible carriers. For example no single PAH molecule has been identified yet. The dust formation is not well understood. What happens to the amorphous carbon dust, observed in the DISM via the bands at $3.4 \mu \mathrm{m}$ and $6.85 \mu \mathrm{m}$ bands, when it is incorporated into the dense ISM is enigmatic. The coupling of the dust and the gas is a vast subject, difficult to model and to study experimentally. All this illustrates that the study of dust in our Galaxy and in external galaxies is a vast and rich subject on which a lot of work has to be done observationally, theoretically and experimentally.

\section{References}

[1] Désert F.X. et al., A\&A 237, (1990) 215-236

[2] Colangeli L. et al., A\&ARv 97, (2003) 97-152

[3] Compiègne M. et al., A\&A 491, (2008) 797-807

[4] Molster F. et al., A\&A 382, (2002) 222-240

[5] Molster F. \& Kemper C., SSRv 119, (2005) 3-28

[6] Tamanai A. et al. A\&A, 501, (2010), 251-267

[7] Voshchinnikov N. \& Henning Th., A\&A 517, (2010)

[8] Keller L. et al. , Nature 417, (2002), 148-150

[9] Dartois E., Cosmic Dust - Near and Far (ASPC Series, 2009) 414 411-427

[10] Gibb E., ApJ 536, (2000) 347-356

[11] Pontoppidan K. et al., A\&A 408, (2003) 981-1007

[12] Pontoppidan K. et al., ApJ 678, (2008) 1005-1031

[13] Smith R.. et al., ApJ, 334, (1988) 209-219

[14] Dartois E. et al., A\&A, 394, (2002) 1057-1068

[15] Dartois E. et al., A\&A, 351, (1999) 1066-1074

[16] Gibb E.L., Whittet D.C.B., Boogert A.C.A. et al., ApJSS, 151, (2004) 35-73

[17] Dartois E. et al., A\&A, 423, (2004) L33-L36

[18] Boersma C. et al., ApJ 690, (2009) 1208-1221

[19] Sellgren K., et al. ApJ 299, (1985) 416-423

[20] Draine B.T., Cosmic Dust - Near and Far (ASPC Series, 2009) 414 453-472

[21] Tielens A.G.G.M., ARA\&A, 46, (2008), 289-337 
[22] Calzetti D., PAHs and the Universe (EAS Publication Series, 2010)

[23] Verstraete L., PAHs and the Universe (EAS Publication Series, 2010)

[24] Iglesias-Groth S., et al., MNRAS 407, (2010) 2157-2165

[25] Iglesias-Groth S., et al., ApJ 685, (2008) L55-L58

[26] Mulas G. et al., A\&A 460, (2006) 93-104

[27] Duley W.W. \& Williams D.A., MNRAS, 205, (1983) 67-

[28] Dartois E. et al., A\&A, 476, (2007) 1235-1242

[29] Joblin C. et al., ApJ, 458, (1996) 610-620

[30] Duley W.W. et al., ApJ, 626, (2005) 933-939

[31] Chiar J. et al., ApJ, 651, (2006) 268-271

[32] Dartois E., et al., A\&A, 432, (2005) 895-908

[33] Nagahara H. et al., Cosmic Dust - Near and Far (ASPCS, 2009) 41443

[34] Decin L., A\&A 516, (2010) A69

[35] Gail H.P \& Sedlmayr E., A\&A 206, (1988) 153-

[36] Patzer A. et al., A\&A 337, (1998) ***-

[37] Dirks U. et al., A\&A 491, (2008) 643-662

[38] Nuth J.A. \& Donn B. et al., JGR 88, (1983) A847-A852

[39] Toppani A. et al., GeCoA 70, (2006) 5035-5060

[40] Nagahara H. et al., Cosmic Dust - Near and Far (ASPCS, 2009) 414 403-410

[41] Cherchneff I. \& Cau P., Asymptotic Giant Branch Stars (IAU Symposium \# 191, 1999), 251-

[42] Allain T., A\&A 323, (1997) 163-176

[43] Frenklach M. \& Feigelson E. D., ApJ 341, (1989) 372

[44] Sabbah H., Bienner L., Klippenstein J. et al., J. Phys. Chem. Lett. 1, (2010) 2962-2967

[45] Cherchneff I. et al., ApJ 401, (1992) 269-287

[46] Cau P., A\&A 392, (2002) 203-213

[47] Jones A.P., et al., ApJ 469, (1996) 740

[48] Dwek E. \& Scalo J., ApJ 239, (1980) 193-211

[49] Draine B., The evolution of the interstellar medium (ASPC Series, 1990), 193-205

[50] Stepnik B. et al., A\&A, 398, (2003) 551-563

[51] Demyk K. et al., A\&A, 368, (2001) L38

[52] Shen C. et al., A\&A 415, (2004) 203-215

[53] Allamandola L, Icarus, 76, (1988) 225-252

[54] Moore M. et al., JGR, 96, (1991) 17 541-17 545

[55] Garrod R.T. \& Herbst E., ApJ, 457, (2006) 927-936

[56] Gerakines P. et al., JGR, 106, (2001) 33 381-33 385

[57] Bennett C. \& Kaiser R., ApJ, 661, (2007) 899-909

[58] Bennett C. \& Kaiser R., ApJ, 660, (2007) 1289-1295

[59] Oberg K. et al., ApJ, 693, (2009) 1209-1218

[60] Zhou L. et al., ApJ, 686, (2008) 1493-1502

[61] Bossa J.B., Theul P., Duvernay F. et al. A\&A 492, (2008) 719-724

[62] Dulieu F. Amiaud L. Congiu E. et al., A\&A, 512, (2010) A30

[63] Miyauchi N. et al., Chem. Phys. Lett., 456, (2008) 27

[64] Ioppolo S. et al., ApJ, 686, (2008) 1474

[65] Dulieu F. et al., A\&A, 512, (2010) A30

[66] Watanabe N. \& Kouchi A., PSSRv, 83, (2008) 439-489

[67] Bisschop S. et al., A\&A 474, (2007) 1061-1071

[68] Watanabe N. et al., ApJ, 668, (2007) 1001-1011

[69] Geppert., Chemical Evolution of the Universe (Faraday discussions, 2006), 133, 177

[70] Hiraoka K. et al., ApJ 577, (2002) 265

[71] Watanabe N. et al., ApJ 616, (2004) 638 
[72] Pontoppidan K et al., A\&A 426, (2004) 925

[73] Fuchs G. et al., A\&A 505, (2009) 629-639

[74] Bossa J. et al. ApJ 707, (2009) 1524-1532

[75] Munoz Caro G. \& Dartois E., A\&A 494, (2009) 109-115

[76] Green, S.D., Bolina A.S., Chen R. et al. MNRAS 398, (2009) 357-367

[77] Acharyya K., Fuchs G.W., Frase H.J., et al. A\&A 466, (2007) 1005-1012

[78] Iglesias E., ApJ, 218, (1977) 697-715

[79] Oberg K. et al., A\&A, 504, (2009) 891-913

[80] Oberg K. et al., A\&A, 496, (2009) 281-293

[81] Schutte W. et al., A\&A, 244, (1991) 190-204

[82] PAHs and the Universe (ASPC Series, 2011)

[83] Pety J. et al., A\&A, 435, (2005) 885-899

[84] Jochims H.W. et al., ApJ, 420, (1994) 307-317

[85] Joblin C., (SF2A, 2003)

[86] Simon A. \& Joblin C. , J. Phys. Chem, 113, (2009) 4878-4888

[87] Brechignac Ph. et al., A\&A, 442, (2005) 239-247

[88] Schmidt M. et al., Int. J. Mass. Spectrom., 252, (2006) 173-179

[89] Cesarsky D. et al., A\&A, 354, (2000) L87-L91

[90] Berné O. et al., A\&A, 469, (2007) 575-586

[91] Berné O. et al., A\&A, 495, (2009) 827-835

[92] Pilleri P. et al., submitted to A\&A

[93] Rapacioli M. et al., A\&A, 429, (2005) 193-204

[94] Rapacioli M. et al., A\&A, 460, (2006) 519-531

[95] Peeters, E. et al., A\&A, 390, (2002) 1089-1113

[96] Bernstein, M. et al., ApJ, 626, (2005) 909-918

[97] Avaro- Galu H et al., A\&A, 517, (2010) A15

[98] Sloan et al. ApJ , 664, (2007) 1144-1153

[99] Pino Th. et al. A\&A, 490, (2008) 665-672

[100] Joblin C. et al., A\&A, 490, (2008) 189-196

[101] Kemper F. et al., ApJ, 609, (2004) 826-837

[102] Kemper F. et al., ApJ, 633, (2005) 534-534

[103] Jäger C. et al., A\&A 401, (2003) 57-65

[104] Brucato J.R. et al., A\&A 413, (2004) 395-401

[105] Demyk K. et al., A\&A 420, (2004) 233-243

[106] O'Donnel J. \& Mathis J., ApJ, 479, (1997) 806

[107] Hirashita H. \& Yan H., MNRAS, 397, (2009) 1061-1074

[108] Boekel R. et al., A\&A 400, (2003) L21-L24

[109] Malfait K. et al., A\&A 332, (1998) L25

[110] Gail H-P., A\&A 378, (2001) 192-213

[111] Gail H-P., A\&A 413, (2004) 571-591

[112] Wooden D. et al., Protostars \& Planets, (University of Arizona Press, 2007) 815

[113] Henning Th. \& Meeus G., To be published in "Physical Processes in Circumstellar Disks around Young Stars", Garcia, PJV (Ed.), Theoretical Astrophysics Series, Chicago University Press 